\title{
Construction of Clinical Biobanks and the Medical Ethics
}

\author{
Meng Yu ${ }^{1, a}$, Qingyu Yan ${ }^{1, a}$, Chi Zhang ${ }^{1}$, Feixiang Wu ${ }^{1}$, Chen Du ${ }^{2 *}$, Weihua Song ${ }^{{ }^{*}}$ \\ ${ }^{1}$ Affiliated Hospital of Hebei University, Baoding 071000, China. E-mail: songweihua_junius@foxmail.com \\ ${ }^{2}$ Baoding Children's Hospital, Baoding 071000, China. E-mail: 393250949@qq.com
}

\begin{abstract}
Nowadays, various types and forms of clinical biobanks have been gradually established worldwide, which have become one of the important components and research platforms of life science and related disease researches in the medical system. This article mainly introduces the construction, management and operation of clinical biobanks, and discusses the medical ethics faced by it.
\end{abstract}

Keywords: Clinical Cases; Medical Ethics; Biobank

\section{Introduction}

Clinical biological samples are of scarcity and non-renewal, the research results and conclusions of which accord with the actual situation of human pathology, effectively reflect the characteristics of the disease, and can provide reference to clinical diagnosis and treatment. In addition, clinical biological samples have become a valuable resource to explore the pathogenesis of the disease, master the law of disease development, develop medical means, and research and develop pharmacy ${ }^{[1]}$.

\section{Establishment, operation and management of clinical biobanks}

The establishment, operation and management of clinical biobanks should be scientific, systematic and comprehensive. Clinical samples of patients to be collected include body fluids, blood, pathological tissues, nucleic acids and proteins. The following will introduce several requirements for the establishment of clinical biobanks should meet the following requirements: 1. In order to ensure the updating and replenishment of samples, stable sample sources are needed. 2. Standardized collection process of biological samples and sound clinical diagnosis and treatment conditions are needed to ensure the timeliness and reliability of samples. 3. Laboratory personnel are required to possess clinical medical knowledge to perfectly take up this job. 4 . Perfect medical facilities and software systems for sample information management are needed. 5. The follow-up processing technology of the samples is necessary to ensure that they can be used in the research of cell biology and molecular biology.

The increasing sample amount is followed by gradual increase of sample information, both of which are under dynamic changes. Therefore, it is an important process to establish a safe, reliable, stable and efficient biobank management system with complete functions for collecting and inducing information of biological samples, so as to ensure the establishment and management of biobanks. Registration of the biological sample information consists of the

a: these authors contributed equally to this work 
following three aspects: first, the basic information of biological samples, such as number, type, amount, date, location, storage location, usage time, situation, personnel, and research. Secondly, information of sample patients, such as citizen information, medical history, family genetic information, diagnosis and treatment indicators and measures, and follow-up information. Finally, the experimental results and feedback of data, such as analysis, collection, follow-up, sample allocation and cooperation of samples, correlation research, and research topics, to reduce repetitive work.

In the application of samples, there should be standard and perfect management measures for the clinical biobanks to prevent the occurrence of unexpected situations such as lost samples and pollution on samples caused by improper management or practices. In order to meet the needs of repeated use, samples should be sub-packed to ensure their survival, and then a scientific and reasonable storage system for biological samples should be established, as well as the follow-up processing and verification technologies, such as cell separation, cell identification, cell detection and nucleic acid extraction technologies. Through that, it can ensure the quality of the extracted products to be provided to the experimenters. Regular sampling inspection should be carried out to the stored biological samples so as to ensure their activity, reduce the loss and improve the work efficiency, thus achieving the purpose of biological research.

The clinical biological sample resources should be carefully protected and rationally utilized, combining the disease of patients, diagnosis and treatment results, routine detection and treatment methods. Specifically speaking, it is necessary to observe and study the occurrence and development of diseases, and comprehensively evaluate the different levels of organs, tissues, cells and molecules; adopting new medical technologies in difficult diagnosed cases is helpful to improve medical quality and accumulate medical experience. Moreover, a large number of confirmed samples of single and multiple diseases are provided for medical research and teaching, which can reduce research time and is benefit to carry out targeted research work.

\section{The medical ethics faced by the clinical biobanks}

In the past, researchers often needed to select and test clinical samples according to the research subject. However, the collected samples were only used for research. The collection and preservation of biological samples ended along with the completion of the subject. Both researchers and sample providers often treat the remaining samples as medical waste after clinical diagnosis and treatment. In the process of collecting clinical cases and operation of biobanks, it is easy to ignore the review and constraints of medical ethics ${ }^{[2]}$.

During collecting samples and running and managing biobanks, the medical ethics involved include the following points: 1 . Whether providers agrees to the collection of samples and related information, and the scope and specific contents agreed by providers; 2 . When samples are taken in the biobanks, whether the sample providers have the right of personal ownership, the right to be informed about the research, and whether the research results and conclusions are fed back to providers. 3. The direction of sample research, researchers and research content should be clear and whether providers still need to cooperate in the process of research. 4 . Whether sample providers can benefit and how to benefit more patients. 5. Whether sample providers will be affected by providing samples and whether the samples will be used for commercial purposes. 6. What measures can be taken to ensure the privacy and safety of providers and samples. 7. How to dispose and destruct samples after the research ${ }^{[3,4]}$.

As a public resource for medical research, biological samples inevitably involve interests. Although the collection of biological samples is different from the clinical research carried out in human body, its essence is human body research and involves the personal interests of the samples providers. Therefore, the principles of medical ethics in clinical research also apply to the ethical review of biobanks.

\section{Ethical review points of clinical biobanks}

\subsection{The benefits and risks of biobanks, and the determination of the ownership}




\section{of biological samples}

The research results of biobanks have improved the medical level of a certain disease, and promoted the development of medicine, by adopting the group research method instead of individual experiments. There is a remarkable difference between in vitro study of biological samples and direct interventional clinical study of human body, and there is almost no damage to sample providers. When samples and their information are included in the biobanks, the individual attributes of these samples are transformed into disease types or group biological attributes of sample types to a certain extent. Therefore, the individual samples become public scientific research resources, thus achieving the sharing of the individual interests of sample providers and the common interests of society.

\subsection{Protect the privacy of biological sample providers and the safety of the samples}

In the actual operation of biobanks, in order to prevent the privacy of sample providers from being disclosed, and ensure the objectivity and accuracy of the research, the unique coded identification is often adopted to encrypt the samples, and the anonymity is applied to protect the privacy of sample providers, thus ensuring that sample providers are not affected by the research ${ }^{[5]}$. At the same time, different levels of access rights should be established so as to clarify the relevant information of the visitors. When external or foreign organizations are involved, corresponding protection measures need to be formulated to prevent the information of clinical biological samples and sample providers from disclosure, which may cause loss of disease information and ethnic heritage information.

\subsection{Set the contents of the informed consent of biobanks}

The basic principles of informed consent are fully informed, fully understanding, independently choosing, personally deciding, etc., while the contents of which include signed consent and oral consent. When designing and defining the contents of the informed consent, it is necessary to fully inform the functions of biobanks and the obligations of the researchers, specify the risks and possible accidents, and emphasize that sample providers have the right to terminate the informed consent at any time. In addition, it is also necessary to set up provisions to restrict researchers, including the qualification level of the personnel in biobanks and the supervision contents for the supervision department, so as to ensure that biological samples are only used for medical research and are destroyed and processed after the research is completed, and ensure that the personal information and privacy of sample providers will not be disclosed. This is not only conducive to the protection of the rights and interests of sample providers, but also can improve the participation of sample providers, as well as protect the researchers. On the basis of full understanding and acceptance of the contents of the informed consent, sample researchers independently choose to sign it and are authorized to use the biological samples and related information under the review of the ethics committee ${ }^{[6,7]}$.

\section{Conclusion}

This article analyzes the establishment, operation and management of clinical biobanks, then briefly expounds the medical ethics faced by the research of clinical biobanks, and finally summarizes the key points of ethical review. In general, to establish, operate and manage biobanks, it is necessary to set up the requirements of collection and induction, classification, data collation, security, and so on, improve the control and management system, and give effective protection to biological samples and their information. With the continuous in-depth work, a system of resource development and utilization of biological samples has been gradually established to provide help, cooperation and mutual assistance for subsequent medical research, and finally the sharing of biological samples can be realized, thus promoting the sustainable development of biobanks ${ }^{[8]}$.

\section{Acknowledgement}

This article is supported by the fund of Affiliated Hospital of Hebei University (No. 2019Q001).

\section{References}

1. Guo Y. Clinical biobank (in Chinese). Beijing: Science Press; 2014. p. 260.

2. Cambon-Thomsen A, Rial-Sebbag E, Knoppers BM. 
Trends in ethical and legal frameworks for the use of human biobanks. European Respiratory Journal 2007; 30(2): 373-382. doi: 10.1183/09031936.00165006.

3. Pawlikowski J, Sak J, Marczewski K. Special report biobank research and ethics: The problem of informed consent in polish biobanks. Archives of Medical Science 2011; 7(5): 896-901. doi: 10.5114/aoms.2011.25568.

4. Liu M. Introduction to biobank and ethics (in Chinese). Chinese Bulletin of Life Sciences 2012; 24(11): 1318-1324.

5. Zhu S, Shen M, Qiu X, et al. Ethical management guidelines for the Shanghai disease-based biobank network. Biopreservation \& Biobanking 2015;
13(1): 8-12. doi: 10.1089/bio.2014.0087.

6. Kaufman D, Bollinger J, Dvoskin $\mathrm{R}$, et al. Preferences for opt-in and opt-out enrollment and consent models in biobank research: A national survey of Veterans Administration patients. Genetics in Medicine 2012; 14(9): 787-794. doi: 10.1038/gim.2012.45.

7. Li $\mathrm{H}$, Liang $\mathrm{Z}$, Zeng $\mathrm{L}$, et al. Ethical issues caused by informed consent signing in the research of bioinformatics database (in Chinese). World Science and Technology 2013; 15(4): 729-734.

8. Xiong N, Liu H, Li Y, et al. Guidelines for ethical review of biomedical research involving humans. Beijing: Science Press; 2014. p. 157. 